\title{
PASADO, PRESENTE Y FUTURO DE LA FORMACIÓN DE MAESTROS EN ESPAÑA
}

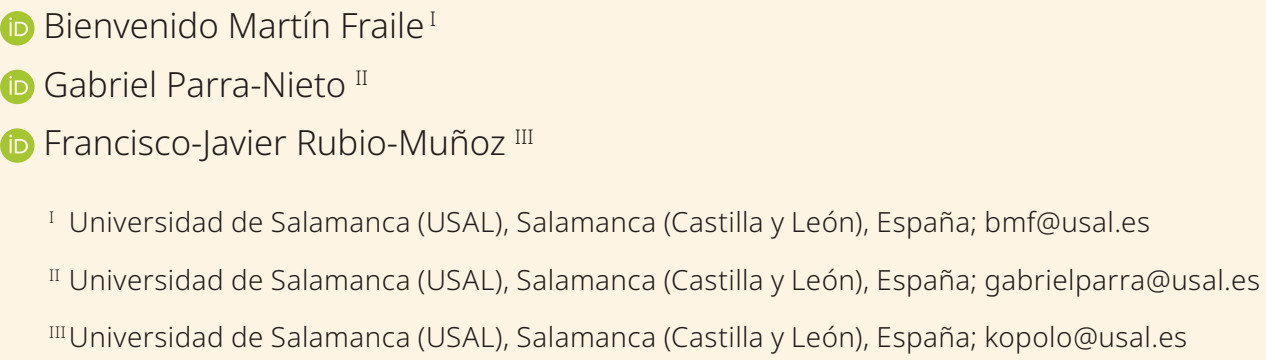

\section{Resumen}

La investigación tiene como objetivo mostrar las claves que han guiado la actuación del docente en el pasado, las que se manifiestan en el presente por medio de la experiencia del maestro y las propuestas que deben guiar el futuro de la profesión. Para ello, se ha realizado un doble análisis cualitativo y cuantitativo a una muestra de 658 docentes entrevistados, cuyo ejercicio profesional tuvo lugar en varias regiones del noroeste de España. El análisis de frecuencias, mediante las tablas de contingencia ofrece datos sobre competencias profesionales y formación del docente. Los resultados indican diferencias en torno al sexo y la edad que se explican por el contexto histórico y educativo previo y posterior al franquismo, así como los cambios introducidos a lo largo de la democracia.

FORMACIÓN DE DOCENTES • COMPETENCIAS • FORMACIÓN INICIAL • FORMACIÓN CONTINUA

\section{PASSADO, PRESENTE E FUTURO DA FORMAÇÃO DE PROFESSORES NA ESPANHA Resumo}

A pesquisa tem como objetivo mostrar as chaves que têm guiado o desempenho do docente no passado, as que se manifestam no presente por meio da experiência do professor e as propostas que devem orientar o futuro da profissão. Para isso, tem se realizado uma dupla análise qualitativa e quantitativa em uma amostra de 658 docentes entrevistados, cujo exercício profissional aconteceu em várias regiões do noroeste da Espanha. A análise de frequências, mediante as tabelas de contingência, oferece dados sobre competências profissionais e formação do docente. Os resultados indicam diferenças em torno do gênero e da idade que se explanam pelo contexto histórico e educacional prévio e posterior ao franquismo, assim como pelas mudanças introduzidas ao longo da democracia. 


\section{PAST, PRESENT AND FUTURE OF TEACHER TRAINING IN SPAIN}

\section{Abstract}

This study seeks to unravel key aspects that have guided teachers' work in the past, those expressed in the present through their classroom experiences, and proposals that should guide the future of the profession. This has involved both qualitative and quantitative analyses based on interviews with 658 teachers working in some regions of northwestern Spain. An analysis of frequencies using contingency tables provides data on professional competencies and teacher training. The results reveal differences in terms of sex and age, explained by the historical and educational context before and after Franco's dictatorship, as well as the significant changes brought about by Spain's transition to democracy.

TEACHING EDUCATION • COMPETENCIES • INITIAL TRAINING • LIFELONG LEARNING

\section{PASSÉ, PRÉSENT ET FUTUR DE LA FORMATION ENSEIGNANTE EN ESPAGNE Résumé}

Cette recherche vise à fournir les éléments-clés ayant déterminé la performance des enseignants dans le passé, ceux qui se dégagent dans le présent, appuyés sur l'expérience de ces professionnels, aussi bien que les propositions qui devraient guider l'avenir de la profession. Pour cela, une analyse qualitative et quantitative a été menée auprès d'un échantillon de 658 enseignants exerçant leur profession dans différentes régions du nord-ouest de l'Espagne. À partir de tableaux de contingence, l'analyse des fréquences a permis de récolter des données sur les compétences professionnelles et la formation des enseignants. Les résultats indiquent des différences en fonction du sexe et de l'âge qui s'expliquent par les contextes historiques et éducatifs avant et après le franquisme, ainsi que par les changements introduits au cours de la démocratie.

FORMATION DES ENSEIGNANTS • COMPÉTENCE • FORMATION INITIALE • FORMATION CONTINUE 
histórica en los actuales temarios destinados a la formación de los futuros docentes. Nuestros alumnos de los grados de magisterio (infantil, primaria o doble grado infantil-primaria) al cabo de cuatro cursos -en el caso de un solo Grado- o cinco -si realizan el Doble Grado- obtienen su título tras haber superado distintas disciplinas curriculares, culturales y profesionales, sin que ninguna de ellas haya hecho alusión a la docencia como profesión con trayectoria secular. Sí aparecen en los últimos años dentro del panorama docente publicaciones destinadas a los maestros en formación y en ejercicio que les acercan un planteamiento de la profesión centrada en la adquisición de competencias - cognitivas, procedimentales/instrumentales y actitudinales - para quienes se acercan a esta actividad profesional. Sin embargo, es notable la ausencia de una perspectiva histórica del tratamiento de esta profesión. Esta circunstancia ha motivado la realización de esta investigación con la intención de permitir a nuestros futuros profesores obtener las claves que han guiado la formación y la práctica docente en el pasado, las que se manifiestan en el presente y las que han de guiar el futuro de la profesión.

En la sociedad del conocimiento, “. . . la calidad del sistema educativo y la formación del profesorado se convierten en una pieza clave, pues para mejorar el desarrollo económico necesitamos mantener un sistema educativo que forme a personas con un alto nivel científico y técnico" (Esteve, 2014, p. 20). Siguiendo este planteamiento, el objetivo general de esta investigación reside en analizar testimonios docentes de maestros jubilados sobre las características de la profesión de maestro en el siglo XXI con el fin de extraer las competencias profesionales y las necesidades formativas prioritarias que los propios docentes consideran más necesarias de cara a habilitar al futuro maestro para una práctica educativa satisfactoria en la actualidad.

Además, se perseguirán dos objetivos específicos: comprobar si existen diferencias en la percepción de la profesión de maestro tanto entre ambos sexos como entre los participantes nacidos antes y después de 1948, fecha que marca diferencias significativas en los elementos analizados.

La originalidad de este trabajo consiste precisamente en abordar el estudio de los testimonios docentes, algo inédito hasta hoy, mediante el tratamiento historiográfico y estadístico de estos documentos donde se recoge la huella de la vida profesional docente de buena parte del siglo XX español y que abre caminos y nuevas vías en la investigación de la cultura escolar (Brady, 2020).

Para la realización de esta propuesta partimos de la consideración de la experiencia docente, la praxis, como un elemento configurativo de la cultura escolar. Para ello contamos con diversas fuentes escritas y orales entre las que destacan las entrevistas realizadas a maestros jubilados sobre su experiencia profesional, de cuya conservación se encarga el Centro Museo Pedagógico de la Universidad de Salamanca (CeMuPe).

\section{Antecedentes y fundamentación teórica}

\section{Pasado de la profesión de maestro: Ia instrucción pública}

El sistema educativo nacional inicia su andadura con la aparición del título IX de la Constitución de Cádiz, en donde emerge por primera vez un concepto que trastoca definitivamente el aparato escolar precedente: la instrucción es pública. Con ello se permite, por un lado, el acceso la educación a todas las capas sociales y, por otro, se genera un interés por ofrecer a los maestros una preparación acorde con sus responsabilidades. Empieza, de esta manera, una nueva fase que rompe con la anterior y en la que se ofrece a los maestros una formación inicial y uniforme para todo el país en 
centros creados específicamente para ello y donde puedan adquirir los conocimientos culturales y las destrezas pedagógicas necesarias para ejercer la profesión con garantía.

Liberales como Pablo Montesino y Gil de Zárate iniciarán la formación de este colectivo de manera institucional, creando para ello el Seminario Central de Maestros del Reino en Madrid en 1839, y posteriormente las Escuelas Normales en todas las provincias de España. En estos centros normalistas, los futuros maestros adquirían competencias culturales, pedagógicas y prácticas. No obstante, las competencias culturales predominaron sobre las demás, tal y como ha quedado demostrado en varios estudios realizados (Luzón Trujillo \& Montes Moreno, 2018).

La Ley Moyano de 1857 traerá importantes novedades en la formación de los maestros. Por un lado, crea la Escuela Normal Central en Madrid para formar a los profesores de las Normales y a los inspectores de enseñanza primaria. Por otro, determina el surgimiento de dos tipos de Escuelas Normales, una de carácter elemental con dos años de formación y otra superior con tres años, otorgando dos títulos para ocuparse de los dos grados de la enseñanza primaria, también dividida entre elemental y superior (Vega Gil, 1988). En cuanto a la cualificación profesional nos encontramos que estos centros normalistas se ocupan más de implantar un currículum cultural que de ofrecer la preparación pedagógica que se les demandaba.

Tendremos que esperar al periodo de la Restauración borbónica para encontrar aires renovadores que impulsen el interés pedagógico de la mano de regeneracionistas e institucionistas. Destacamos entre todas las innovaciones la creación de la Escuela Superior del Magisterio para formar profesores de las Escuelas Normales y a inspectores de primera enseñanza. Esta Escuela tendrá como misión principal formar maestros cultos y de vocación, donde la formación pedagógica y el carácter universitario se impulsen, para llevar a cabo la difusión de la educación primaria por todo el país. Asimismo, este Centro será la puerta por donde entren a España las ideas y propuestas prácticas del Movimiento de la Escuela Nueva.

Con la proclamación de la Segunda República, la formación de maestros alcanza un gran desarrollo mediante el Plan Profesional de 1931. Un plan configurado a distintos niveles: cultural, profesional y práctico mediante el acceso desde el bachillerato que aporta el nivel cultural necesario y ocupándose de la formación profesional las propias Escuelas Normales por medio del estudio de la psicología y pedagogía, dejando la propuesta práctica para las escuelas de primaria (Martín Fraile, 2015).

Habrá que esperar hasta 1945 con la publicación de la Ley de Educación Primaria para comprobar de hecho la influencia del nuevo régimen político salido de la Guerra Civil. Mediante esta ley, se incorpora a las Escuelas Normales la ideología que representa el binomio patria-religión tras la ruptura con los planteamientos republicanos, abriéndose un escenario de retroceso en la formación los maestros (Martín Fraile, 2017). Hasta la llegada de los tecnócratas al gobierno no se impulsan cambios significativos que reflejen el empuje de la economía sobre el sistema educativo. Esta influencia marca el nacimiento de un nuevo plan de estudios, el plan de 1967, que inicia el despertar de la formación normalista, fomentando el estudio de la pedagogía y abriendo la capacitación profesional por medio de las didácticas especiales.

A la postre resultará un plan de escasa vigencia ya que en pocos años se promulga la Ley General de Educación de 1970 que pasa de una concepción del maestro más técnica en lugar de carismática (Mainer Baqué \& Mateos Monero, 2011), y entre otros avances presenta la incorporación de los estudios de magisterio a los espacios universitarios, tal y como se recoge en el Plan de 1971.

Tras la Constitución de 1978, la llegada de nuevo de la democracia irrumpirá con fuerza en el sistema educativo promulgando en 1990 una nueva ley de educación, la Ley de Ordenación General del Sistema Educativo, LOGSE. De esta ley se deriva el plan de 1990, que impulsa la creación de las Facultades de Educación donde las universidades cobijarán los estudios de formación de maestros, con un nivel mayor de exigencia y nuevas especialidades para afrontar las demandas sociales. Plan de poca 
vigencia ya que el movimiento por conseguir alcanzar un carácter más profesional y de calidad a los maestros provoca una profunda reestructuración al surgir en 2004 la propuesta de los nuevos Grados de maestros, infantil y primaria, comenzando en 2010 su andadura los nuevos títulos tras cuatro años de formación simultánea, teórica y práctica.

\section{Presente de la profesión del maestro: formación inicial y continua}

El Espacio Europeo de Educación Superior ha adaptado a los retos actuales la formación y los sistemas de selección del profesorado, si bien organismos internacionales como la UNESCO, la OCDE o la propia UE no se muestran demasiado explícitos a la hora de establecer un mecanismo de acceso a la profesión docente; tan sólo intencionalidades generalistas. Con todo puede decirse que existe un modelo de capacitación competencial profesional, práctica e investigadora (Valle, 2012; Álvarez-López, 2015). En este modelo se enfrentan dos concepciones en la formación del profesorado: uno profesionalizante y otro pragmático, sin reflexión epistemológica (Prats, 2016).

El reflejo de este contexto en nuestras fronteras supuso el nacimiento de la LOGSE en 1990, un choque frontal con respecto a la LGE de 1970, de carácter tecnocrático. La nueva ley planteaba un modelo reflexivo sobre la práctica, donde el profesor es un investigador en el aula y donde prima la reflexión sobre la acción. Sin embargo, en el campo de la formación docente se obtuvieron resultados modestos, revelándose dos tipos de problemas: el modo y el modelo de formación adoptado, que deberían buscar una mayor interacción equilibrada de todo el sistema educativo, y el desfase entre las reformas de la formación inicial y la propia profesión, ya que no se elevó la calidad del trabajo docente, a quienes, además, les faltan incentivos en diversos ámbitos: formación continua, trabajo en equipo, etc. (Tiana, 2013).

En cualquier caso, hay un acuerdo general en distinguir entre la formación inicial y la formación continua dentro de las etapas en la formación de los docentes. Sin embargo, bastantes autores se han percatado de la poca coherencia entre la formación inicial y la continua de los profesores (Cantón-Mayo et al., 2013; Valle \& Manso, 2014), ya que ambas deberían entenderse como una formación ininterrumpida con oportunidades de aprendizaje formal, informal y no formal. De esta disfunción también son conscientes las instituciones y organismos educativos. El XXI Encuentro de Consejos Escolares Autonómicos en 2015, puso de relieve las necesidades en los dos ámbitos de su formación: inicial, construyendo una identidad profesional definida y confeccionando un programa que facilite una sólida preparación; continua, como un derecho y un deber del profesorado, persiguiendo la actualización y adaptación a los cambios sociales, además de proporcionar a los docentes entornos compartidos de reflexión y aprendizaje. Así mismo, en 2018 la Conferencia de Decanos/as y Directores/ as de Centros de Educación expresaba la necesidad de un marco que garantice una carrera profesional, siendo evidente la continuidad entre la formación y el acceso a la profesión docente.

Esta búsqueda de consenso en torno a un perfil de competencias profesionales docentes que dé continuidad y coherencia a ambas formaciones, inicial y continua, no puede comprenderse sin las competencias en TIC. Éstas son demandadas por la sociedad actual como parte indispensable y transversal tanto de la formación inicial como continua de los futuros maestros. Sin embargo, estudios recientes parecen indicar que los docentes españoles no han desarrollado de forma suficiente la competencia digital, destacando la falta de formación en TIC en las aulas universitarias (Llamas \& Macías, 2018). Paradójicamente, existe una percepción elevada y positiva en cuanto al uso y manejo de las TIC en contraste con el uso didáctico que realizan de las herramientas digitales (Romero-Martín et al., 2017; Tárraga et al., 2017). 


\section{Historias de vida docente}

El interés por la vida interna de la escuela, su cotidianeidad, a través de la microhistoria y la etnografía escolares es bastante reciente (Jaramillo et al., 2020). A mediados de los 90 comienza a manifestarse la importancia de la cultura escolar y su intrahistoria, cuyo conocimiento es favorecido por la creación de museos pedagógicos que recuperan y ponen en valor el patrimonio histórico educativo. En el caso particular del CeMuPe, de la Universidad de Salamanca, su principal objetivo es fomentar la participación de los alumnos en la interpretación de la génesis, evolución y cambios de los procesos educativos a lo largo del tiempo, cumpliendo con la función de interacción con la sociedad.

Una de las líneas de investigación abiertas por el CeMuPe es la cultura oral, centrada en el análisis de lo que transmiten los protagonistas de la escuela: los docentes y el alumnado mediante sus testimonios de vida escolar. De este modo, los testimonios de maestros se convierten en una fuente privilegiada para el estudio de la evolución de la profesión docente desde el siglo XIX, destacando la importancia de la formación, la función educadora y la transmisión de valores (de Gabriel Fernández, 2015).

Martín Fraile (2017) pone de manifiesto la importancia de reivindicar un mayor conocimiento de lo que acontece dentro de las aulas escolares, dando a conocer la experiencia profesional de los maestros y, por tanto, este conocimiento ayudará a dirimir si las prácticas habituales de enseñanza pueden o no mejorarse, y a la vez descubrir qué estrategias utilizadas por el profesorado son las más válidas para mejorar la situación académica y el proceso educativo de sus alumnos.

Por ello, consideramos estos testimonios como un instrumento y una fuente primaria fundamental de investigación. Por medio de ellos podemos llevar a cabo una reconstrucción histórica del significado de esta profesión en la centuria pasada. En este sentido, nuestra investigación se enmarca en un proyecto de mayor envergadura, el de recoger testimonios de maestros que nacieron en la primera mitad del siglo XX.

En definitiva, se pretende proporcionar las claves y pautas para ejercer la profesión docente desde el conocimiento del pasado de los maestros para afrontar los retos del futuro por delante. Ello implica, como sostiene Bolívar (2014), transformar los relatos de vida (life stories) en historias de vida (life histories), lo cual permitirá comprenderlos y situarlos en el contexto sociocultural al que pertenecen.

\section{Método}

La investigación llevada a cabo parte de una muestra de 658 testimonios de docentes y su análisis, siguiendo una estrategia metodológica de tipo cuantitativa y cualitativa, permitió obtener información muy diversa y completa tanto de las competencias profesionales como de las necesidades de formación consideradas como prioritarias para la profesión de maestro en el siglo XXI. Para ello, nada mejor que poner el foco de atención en quienes han dedicado toda su vida laboral al desempeño de esta profesión: docentes jubilados.

\section{Muestra}

Este estudio forma parte de una investigación más amplia que analiza 1500 cuadernos con testimonios y vivencias de docentes jubilados de Educación Infantil o Educación Primaria que han ejercido su profesión en diferentes centros escolares rurales y urbanos de la geografía española, dentro de los fondos pertenecientes al CeMuPe de la Universidad de Salamanca. 
Para esta investigación, se ha utilizado una muestra, recogida en el año 2017, de carácter incidental y no probabilístico. Se compone de 658 testimonios de maestros jubilados que desarrollaron su labor docente en las comunidades de Castilla y León, Extremadura y Asturias. Sus edades están comprendidas entre los 61 y 94 años, siendo la media 71,70 (DT=6,11), de los cuales el 65,8\% son mujeres y el $32,2 \%$, hombres, correspondiendo el restante porcentaje a los datos perdidos (2\%).

Con relación a la distribución en grupos de edad, un 51,2\% nacieron antes de 1948 y un 41,9\% después de este año, correspondiendo el restante porcentaje a los datos perdidos (6,9\%). Como se verá, esta fecha (1948) marca diferencias significativas en los elementos analizados.

En cuanto al análisis de la formación inicial de la muestra (Tabla 1), la mayor parte de los sujetos participantes en esta investigación $(81,3 \%)$ han cursado estudios de Magisterio en sus diferentes modalidades. El $9 \%$ se han formado para ejercer su docencia en la etapa de educación infantil y aproximadamente un 5\% han estudiado otros grados vinculados al ámbito educativo, entre los que destacan los de pedagogía y psicología.

\section{TABLA 1}

FORMACIÓN INICIAL DE LOS PARTICIPANTES EN EL ESTUDIO

\begin{tabular}{|c|c|c|c|c|c}
\hline \multirow{4}{*}{ TITULACIÓN } & Porcentaje & $\begin{array}{c}\text { Porcentaje } \\
\text { válido }\end{array}$ & $\begin{array}{c}\text { Porcentaje } \\
\text { acumulado }\end{array}$ \\
\hline \multirow{4}{*}{ Válido } & Magisterio general & 47,1 & 49,4 & 85,2 & 55,0 \\
\cline { 2 - 6 } & Magisterio primaria & 25,2 & 26,4 & 35,8 & 100,0 \\
\cline { 2 - 6 } & Magisterio infantil & 9,0 & 9,4 & 9,4 & \\
\cline { 2 - 6 } & Magisterio EGB & 9,0 & 9,4 & 94,7 & \\
\cline { 2 - 6 } & Pedagogía & 1,5 & 1,6 & 96,2 & \\
\cline { 2 - 6 } & Psicología & 0,9 & 0,9 & 97,2 & \\
\hline & Otras & 2,7 & 2,8 & 100,0 & \\
\hline Perdidos & Total & 95,5 & 100,0 & & \\
\hline Total & Sistema & 4,5 & & & \\
\hline
\end{tabular}

Fuente: Elaboración propia.

\section{Instrumento}

Para llevar a cabo esta investigación se utilizó una entrevista oral, individual y jerarquizada, centrada en aspectos como los años de docencia, concepción del magisterio, prácticas educativas, función social, formación y perspectiva de la profesión de maestro. Su diseño, elaboración y validación están descritos en otros trabajos de investigación (Martín Fraile, 2015, 2017). La información de las entrevistas, recogida en cuadernillos, refleja una "historia de vida" docente... En esta investigación, se analizaron las respuestas a las preguntas: ¿cuáles deberían ser las características de la profesión de maestro del siglo XXI? y ¿̨cuál debe ser la formación inicial y continua de los maestros?

\section{Procedimiento}

Tras la recogida de los 1500 testimonios que forman parte del Fondo de "Testimonios de la Vida Escolar” del CeMuPe, se seleccionaron aleatoriamente 333 entrevistas con el objetivo de analizar las percepciones acerca de las características profesionales necesarias para el buen ejercicio del magisterio. Además, se seleccionaron 325 entrevistas diferentes para analizar sus percepciones sobre los requisitos formativos que deberían ser necesarios para ser un buen maestro en la actualidad; en total, una muestra de 658 participantes. Posteriormente se llevó a cabo un análisis cualitativo 
de contenido, obteniendo las categorías que se repiten con más frecuencia en cada una de las respuestas de los docentes, dando como resultado la lista que figura en las tablas 2 y 3 .

Las categorías establecidas para analizar la variable "características del docente" se agruparon en siete: vocacional/gusto por los niños, formador de personas/ciudadanos responsables, impulsor de una educación integral, habilidades y competencias sociales, profesionalidad, afán de formación y transmisor de conocimientos y otros. En esta categoría se han incluido aquellos recursos que fueron nombrados por menos del $2 \%$ de toda la muestra. Mientras que las categorías establecidas para el análisis de la variable "requisitos formativos" se agruparon en nueve: necesidad de formación continua, formación en TIC, formación en didáctica y metodología práctica, formación cultural y contenidos, formación psicopedagógica, formación y gestión de centros, formación en valores y aptitudes personales, formación para la atención a la diversidad y formación en mediación familia-escuela.

Una vez establecidas las diferentes categorías se analizaron cuantitativamente los datos con el programa estadístico SPSS 23. Los parámetros descriptivos se llevaron a cabo mediante análisis de frecuencias. Para comprobar si el sexo o el año de nacimiento pueden contribuir a explicar las percepciones de los docentes sobre las características y formación necesaria para ser un buen maestro en el siglo XXI se realizaron tablas de contingencia. Las variables sexo y año de nacimiento serán la variable independiente y las variables "características del maestro" y "necesidades de formación" serán las variables dependientes.

\section{Resultados}

En la Tabla 2 se presentan las frecuencias de las siete categorías más recurrentes en las respuestas de los docentes a la pregunta relativa a las características de la profesión de maestro del siglo XXI y, en la Tabla 3, las frecuencias de las nueve categorías más comunes en las respuestas de los docentes a la pregunta relativa al tipo de formación que consideran que debe tener el maestro.

TABLA 2

CATEGORÍAS MÁS FRECUENTES RESPECTO A LAS CARACTERÍSTICAS QUE DEBE TENER EL MAESTRO

\begin{tabular}{c|c|c}
\hline CARACTERÍSTICAS & Frecuencia & Porcentaje \\
\hline Vocacional/Gusto por los niños & 152 & 45,8 \\
\hline Formador de ciudadanos responsables & 134 & 40,4 \\
\hline Profesionalidad & 130 & 39,2 \\
\hline Impulsor de una Educación Integral & 129 & 38,9 \\
\hline Habilidades y competencias sociales & 109 & 32,8 \\
\hline Afán de formación & 103 & 31,0 \\
\hline Transmisor de conocimientos & 96 & 28,9 \\
\hline Otras & 5 & 1,5 \\
\hline
\end{tabular}

Fuente: Elaboración propia.

El perfil vocacional y de gusto por los niños resulta la categoría más frecuente en la respuesta de los maestros (45,8\%), seguida de la que alude a su función como formador de ciudadanos responsables $(40,4 \%)$. En tercer y cuarto lugar, con una diferencia mínima entre ellas, figura la profesionalidad y la labor del docente como impulsor de una educación integral de los alumnos (39,2\% y 38,9\%). Dentro del rol del maestro también se mencionan la necesidad de fomentar habilidades y competencias sociales en los estudiantes $(32,8 \%)$, la importancia de la formación para llevar a cabo una práctica docente satisfactoria (31\%) y, por último, su función más tradicional como transmisor de conocimientos $(28,9 \%)$. 
TABLA 3

CATEGORÍAS MÁS FRECUENTES RESPECTO A LA FORMACIÓN QUE DEBE TENER EL MAESTRO

\begin{tabular}{|c|c|c|}
\hline FORMACIÓN & Frecuencia & Porcentaje \\
\hline Necesidad de formación continua & 246 & 74,1 \\
\hline Didáctica y metodología práctica & 149 & 34,9 \\
\hline Formación en TIC & 130 & 34,9 \\
\hline Formación cultural y contenidos & 116 & 30,1 \\
\hline Formación psicopedagógica & 100 & 13,9 \\
\hline Formación en valores y aptitudes personales & 46 & 4,8 \\
\hline Formación para la atención a la diversidad & 16 & 3,6 \\
\hline Formación y gestión de centros & 12 & 3,0 \\
\hline Formación en mediación familia-escuela & 10 & 9,9 \\
\hline Otras & 33 & 3 \\
\hline
\end{tabular}

Fuente: Elaboración propia.

Como se puede observar, la necesidad de formación continua resulta ser la categoría más frecuente en las respuestas de los maestros $(74,1 \%)$ seguida de la formación en didáctica y metodología práctica (45\%) y formación en TIC (39,2\%).

La formación cultural y la formación psicopedagógica son mencionadas por un 35\% y un 30\% de la muestra respectivamente. Por el contrario, tener formación en gestión de centros y en situaciones de mediación entre familia y escuela son las menos frecuentes (3,6\% y 3,0\%).

TABLA 4

DIFERENCIAS DE SEXO EN LA PERCEPCIÓN DE LOS MAESTROS SOBRE LAS CARACTERÍSTICAS

\begin{tabular}{|c|c|c|c|c|c|c|c|}
\hline CARACTERÍSTICAS & Sexo & Frecuencia & $\begin{array}{l}\% \text { dentro } \\
\text { sexo }\end{array}$ & $\begin{array}{c}\text { \% dentro de carac- } \\
\text { terísticas }\end{array}$ & Valor & P-valor & Coef. Conting \\
\hline \multirow{2}{*}{$\begin{array}{l}\text { Transmisor de } \\
\text { conocimientos }\end{array}$} & Hombre & 32 & 29,9 & 34,0 & \multirow{2}{*}{0,075} & \multirow{2}{*}{0,784} & \multirow{2}{*}{0,015} \\
\hline & Mujer & 62 & 28,4 & 66,0 & & & \\
\hline \multirow{2}{*}{$\begin{array}{l}\text { Formador de } \\
\text { ciudadanos } \\
\text { responsables }\end{array}$} & Hombre & 42 & 39,3 & 32,1 & \multirow{2}{*}{0,074} & \multirow{2}{*}{0,786} & \multirow{2}{*}{0,015} \\
\hline & Mujer & 89 & 40,8 & 67,9 & & & \\
\hline \multirow{2}{*}{$\begin{array}{l}\text { Vocacional/Gusto por } \\
\text { los niños }\end{array}$} & Hombre & 34 & 31,8 & 23,0 & \multirow{2}{*}{12,183} & \multirow{2}{*}{0,000} & \multirow{2}{*}{0,190} \\
\hline & Mujer & 114 & 52,3 & 77,0 & & & \\
\hline \multirow{2}{*}{ Profesionalidad } & Hombre & 40 & 37,4 & 31,5 & \multirow{2}{*}{0,192} & \multirow{2}{*}{0,661} & \multirow{2}{*}{0,024} \\
\hline & Mujer & 87 & 39,9 & 68,5 & & & \\
\hline \multirow{2}{*}{ Afán de formación } & Hombre & 36 & 33,6 & 35,3 & \multirow{2}{*}{0,378} & \multirow{2}{*}{0,538} & \multirow{2}{*}{0,034} \\
\hline & Mujer & 66 & 30,3 & 64,7 & & & \\
\hline \multirow{2}{*}{$\begin{array}{l}\text { Impulsor de una } \\
\text { educación integral }\end{array}$} & Hombre & 48 & 44,9 & 38,4 & \multirow[t]{2}{*}{2,759} & \multirow[t]{2}{*}{0,097} & \multirow{2}{*}{0,092} \\
\hline & Mujer & 77 & 35,3 & 61,6 & & & \\
\hline \multirow{2}{*}{$\begin{array}{l}\text { Habilidades y } \\
\text { competencias } \\
\text { sociales }\end{array}$} & Hombre & 43 & 40,2 & 40,6 & \multirow[b]{2}{*}{4,161} & \multirow[b]{2}{*}{0,041} & \multirow[b]{2}{*}{0,112} \\
\hline & Mujer & 63 & 28,9 & 59,4 & & & \\
\hline
\end{tabular}

Fuente: Elaboración propia.

Además de la frecuencia y los porcentajes de respuesta positiva dentro del año de nacimiento y dentro de las características y las necesidades de formación, las tablas 4 y 5 contienen el valor experimental (Chi-Cuadrado), el p valor y el coeficiente de contingencia de la prueba para el cruce 
de la variable sexo con las siete categorías establecidas para las características del maestro y las nueve categorías de necesidad de formación definidas como variables, respetivamente.

TABLA 5

DIFERENCIAS DE SEXO EN LA PERCEPCIÓN DE LOS MAESTROS SOBRE NECESIDADES DE FORMACIÓN

\begin{tabular}{|c|c|c|c|c|c|c|c|}
\hline $\begin{array}{l}\text { NECESIDADES DE } \\
\text { FORMACIÓN }\end{array}$ & Sexo & Frecuencia & $\begin{array}{l}\% \text { dentro } \\
\text { sexo }\end{array}$ & $\begin{array}{l}\% \text { dentro de } \\
\text { formac. }\end{array}$ & Valor & P-valor & $\begin{array}{l}\text { Coef. } \\
\text { Conting }\end{array}$ \\
\hline \multirow{2}{*}{ Formación continua } & Hombre & 77 & 72,0 & 31,8 & \multirow{2}{*}{0,52} & \multirow{2}{*}{0,47} & \multirow{2}{*}{0,004} \\
\hline & Mujer & 165 & 75,7 & 68,2 & & & \\
\hline \multirow{2}{*}{ Formación TIC } & Hombre & 42 & 39,3 & 32,8 & \multirow{2}{*}{0,00} & \multirow{2}{*}{0,97} & \multirow{2}{*}{0,00} \\
\hline & Mujer & 86 & 39,4 & 67,2 & & & \\
\hline \multirow{2}{*}{$\begin{array}{l}\text { Formación metodología } \\
\text { práctica }\end{array}$} & Hombre & 49 & 45,8 & 33,8 & \multirow{2}{*}{0,09} & \multirow{2}{*}{0,76} & \multirow{2}{*}{0,02} \\
\hline & Mujer & 96 & 44,0 & 66,2 & & & \\
\hline \multirow{2}{*}{$\begin{array}{l}\text { Formación cultural y } \\
\text { contenidos }\end{array}$} & Hombre & 32 & 29,9 & 28,3 & \multirow{2}{*}{1,66} & \multirow{2}{*}{0,20} & \multirow{2}{*}{0,07} \\
\hline & Mujer & 81 & 37,2 & 71,7 & & & \\
\hline \multirow{2}{*}{ Formación psicopedagógica } & Hombre & 36 & 33,6 & 37,1 & \multirow{2}{*}{1,10} & \multirow{2}{*}{0,29} & \multirow{2}{*}{0,06} \\
\hline & Mujer & 61 & 28,0 & 62,9 & & & \\
\hline \multirow{2}{*}{$\begin{array}{c}\text { Formación gestión de } \\
\text { centros }\end{array}$} & Hombre & 4 & 3,7 & 33,3 & \multirow{2}{*}{0,00} & \multirow{2}{*}{0,97} & \multirow{2}{*}{0,00} \\
\hline & Mujer & 8 & 3,7 & 66,7 & & & \\
\hline \multirow{2}{*}{$\begin{array}{l}\text { Formación valores y } \\
\text { actitudes personales }\end{array}$} & Hombre & 17 & 15,9 & 37,8 & \multirow[b]{2}{*}{0,56} & \multirow[b]{2}{*}{0,45} & \multirow[b]{2}{*}{0,04} \\
\hline & Mujer & 28 & 12,8 & 62,2 & & & \\
\hline \multirow{2}{*}{$\begin{array}{c}\text { Formación atención a la } \\
\text { diversidad }\end{array}$} & Hombre & 6 & 5,6 & 37,5 & \multirow{2}{*}{0,15} & \multirow{2}{*}{0,70} & \multirow{2}{*}{0,02} \\
\hline & Mujer & 10 & 4,6 & 62,5 & & & \\
\hline \multirow{2}{*}{$\begin{array}{l}\text { Formación mediación } \\
\text { familia-escuela }\end{array}$} & Hombre & 4 & 3,7 & 40,0 & \multirow{2}{*}{0,23} & \multirow{2}{*}{0,63} & \multirow{2}{*}{0,03} \\
\hline & Mujer & 6 & 2,8 & 60,0 & & & \\
\hline
\end{tabular}

Fuente: Elaboración propia.

Según el análisis de la Tabla 4, la relación entre el sexo de los sujetos y las variables características del maestro vocacional/gusto por los niños $(\mathrm{P} .=0,000)$ y habilidades y competencias sociales $(\mathrm{P} .=0,041)$ son significativas. Es decir, la predominancia de las características vocacionales y las relacionadas con el desarrollo de las habilidades y competencias sociales es mayor en las mujeres. Para las otras dos variables lo que predomina es el no en el colectivo de mujeres. Sin embargo, la asociación entre el sexo y las otras variables no es muy fuerte como se puede apreciar observando el coeficiente de contingencia. En cambio, tal y como se puede observar en la Tabla 5 -la que hace alusión a las diferencias de sexo en la percepción sobre las necesidades de formación- aunque las frecuencias en las percepciones de hombres y mujeres son diferentes, siendo mayor el porcentaje en las mujeres que en los hombres, las diferencias no llegan a ser significativas. 
TABLA 6

DIFERENCIAS ENTRE MAESTROS NACIDOS ANTES Y DESPUÉS DE 1948 CON RELACIÓN A LAS CARACTERÍSTICAS QUE DEBE TENER EL MAESTRO DEL SIGLO XXI

\begin{tabular}{|c|c|c|c|c|c|c|c|}
\hline CARACTERÍSTICAS & $\begin{array}{l}\text { Grupo de } \\
\text { Edad }\end{array}$ & Frecuencia & $\begin{array}{l}\% \text { dentro } \\
\text { año nac. }\end{array}$ & $\begin{array}{l}\% \text { dentro de } \\
\text { caract. }\end{array}$ & Valor & P-valor & $\begin{array}{l}\text { Coef. } \\
\text { Conting }\end{array}$ \\
\hline \multirow{2}{*}{ Transmisor de conocimientos } & $<1948$ & 43 & 25,3 & 46,2 & \multirow{2}{*}{4,144} & \multirow{2}{*}{0,042} & \multirow{2}{*}{0,115} \\
\hline & $>1948$ & 50 & 36,0 & 53,8 & & & \\
\hline \multirow{2}{*}{$\begin{array}{l}\text { Formador de ciudadanos } \\
\text { responsables }\end{array}$} & $<1948$ & 69 & 40,6 & 53,9 & \multirow{2}{*}{0,109} & \multirow{2}{*}{0,742} & \multirow{2}{*}{0,019} \\
\hline & $>1948$ & 59 & 42,4 & 46,1 & & & \\
\hline \multirow{2}{*}{$\begin{array}{l}\text { Vocacional/Gusto por los } \\
\text { niños }\end{array}$} & $<1948$ & 86 & 50,6 & 61,0 & \multirow{2}{*}{3,743} & \multirow{2}{*}{0,053} & \multirow{2}{*}{0,109} \\
\hline & $>1948$ & 55 & 39,6 & 39,0 & & & \\
\hline \multirow{2}{*}{ Profesionalidad } & $<1948$ & 37 & 21,8 & 30,1 & \multirow{2}{*}{51,336} & \multirow{2}{*}{0,000} & \multirow{2}{*}{0,377} \\
\hline & $>1948$ & 86 & 61,9 & 69,9 & & & \\
\hline \multirow{2}{*}{ Afán de formación } & $<1948$ & 59 & 34,7 & 60,8 & \multirow{2}{*}{1,927} & \multirow{2}{*}{0,165} & \multirow{2}{*}{0,079} \\
\hline & $>1948$ & 38 & 27,3 & 39,2 & & & \\
\hline \multirow{2}{*}{$\begin{array}{c}\text { Impulsor de una educación } \\
\text { integral }\end{array}$} & $<1948$ & 65 & 38,2 & 54,6 & \multirow{2}{*}{0,012} & \multirow{2}{*}{0,912} & \multirow{2}{*}{0,006} \\
\hline & $>1948$ & 54 & 38,8 & 45,4 & & & \\
\hline \multirow{2}{*}{$\begin{array}{l}\text { Habilidades y competencias } \\
\text { sociales }\end{array}$} & $<1948$ & 48 & 28,2 & 47,5 & \multirow{2}{*}{3,403} & \multirow{2}{*}{0,065} & \multirow{2}{*}{0,104} \\
\hline & $>1948$ & 53 & 38,1 & 52,5 & & & \\
\hline
\end{tabular}

Fuente: Elaboración propia.

Por su parte, además de la frecuencia y los porcentajes de respuesta positiva dentro del año de nacimiento y dentro del perfil, las tablas 6 y 7 contienen el valor experimental (Chi-Cuadrado), el p valor y el coeficiente de contingencia de la prueba para el cruce de la variable año de nacimiento (anteriores a 1948 y posteriores) con la variable perfil del maestro y requisitos de formación, respectivamente.

\section{TABLA 7}

DIFERENCIAS ENTRE MAESTROS NACIDOS ANTES Y DESPUÉS DE 1948 CON RELACIÓN A LOS REQUISITOS FORMATIVOS DE UN BUEN MAESTRO

\begin{tabular}{|c|c|c|c|c|c|c|c|}
\hline $\begin{array}{l}\text { REQUISITOS } \\
\text { FORMATIVOS }\end{array}$ & $\begin{array}{c}\text { Grupo de } \\
\text { edad }\end{array}$ & Frecuencia & $\begin{array}{l}\% \text { dentro } \\
\text { año nac. }\end{array}$ & $\begin{array}{l}\% \text { dentro de } \\
\text { formación }\end{array}$ & Valor & P-valor & $\begin{array}{l}\text { Coef. } \\
\text { Conting }\end{array}$ \\
\hline \multirow{2}{*}{ Formación continua } & $<1948$ & 126 & 74,1 & 55,0 & \multirow{2}{*}{0,00} & \multirow{2}{*}{0,99} & \multirow{2}{*}{0,00} \\
\hline & $>1948$ & 103 & 74,1 & 45,0 & & & \\
\hline \multirow{2}{*}{ Formación TIC } & $<1948$ & 49 & 28,8 & 39,5 & \multirow{2}{*}{20,7} & \multirow{2}{*}{0,000} & \multirow{2}{*}{0,25} \\
\hline & $>1948$ & 75 & 54,0 & 60,5 & & & \\
\hline \multirow{2}{*}{$\begin{array}{l}\text { Formación } \\
\text { metodología práctica }\end{array}$} & $<1948$ & 53 & 31,2 & 37,9 & \multirow{2}{*}{0,09} & \multirow{2}{*}{0,000} & \multirow{2}{*}{0,30} \\
\hline & $>1948$ & 87 & 62,6 & 62,1 & & & \\
\hline \multirow{2}{*}{$\begin{array}{l}\text { Formación cultural y } \\
\text { contenidos }\end{array}$} & $<1948$ & 80 & 47,1 & 72,1 & \multirow{2}{*}{20,36} & \multirow{2}{*}{0,000} & \multirow{2}{*}{0,25} \\
\hline & $>1948$ & 31 & 22,3 & 27,9 & & & \\
\hline \multirow{2}{*}{$\begin{array}{c}\text { Formación } \\
\text { psicopedagógica }\end{array}$} & $<1948$ & 33 & 19,4 & 35,1 & \multirow{2}{*}{21,64} & \multirow{2}{*}{0,000} & \multirow{2}{*}{0,26} \\
\hline & $>1948$ & 61 & 43,9 & 64,9 & & & \\
\hline \multirow{2}{*}{$\begin{array}{l}\text { Formación gestión de } \\
\text { centros }\end{array}$} & $<1948$ & 3 & 1,8 & 27,3 & \multirow{2}{*}{3,55} & \multirow{2}{*}{0,06} & \multirow{2}{*}{0,11} \\
\hline & $>1948$ & 8 & 5,8 & 72,7 & & & \\
\hline \multirow{2}{*}{$\begin{array}{l}\text { Formación valores y } \\
\text { actitudes personales }\end{array}$} & $<1948$ & 20 & 11,8 & 47,6 & \multirow{2}{*}{1,07} & \multirow{2}{*}{0,30} & \multirow{2}{*}{0,06} \\
\hline & $>1948$ & 22 & 15,8 & 52,4 & & & \\
\hline
\end{tabular}


(Continuación)

\begin{tabular}{|c|c|c|c|c|c|c|c|}
\hline $\begin{array}{l}\text { REQUISITOS } \\
\text { FORMATIVOS }\end{array}$ & $\begin{array}{l}\text { Grupo de } \\
\text { edad }\end{array}$ & Frecuencia & $\begin{array}{l}\text { \% dentro } \\
\text { año nac. }\end{array}$ & $\begin{array}{l}\text { \% dentro de } \\
\text { formación }\end{array}$ & Valor & P-valor & $\begin{array}{l}\text { Coef. } \\
\text { Conting }\end{array}$ \\
\hline \multirow{2}{*}{$\begin{array}{c}\text { Formación atención a } \\
\text { la diversidad }\end{array}$} & $<1948$ & 5 & 3,0 & 31,3 & \multirow{2}{*}{3,80} & \multirow{2}{*}{$0,05^{*}$} & \multirow{2}{*}{0,11} \\
\hline & $>1948$ & 11 & 7,9 & 68,8 & & & \\
\hline \multirow{2}{*}{$\begin{array}{l}\text { Formación mediación } \\
\text { familia-escuela }\end{array}$} & $<1948$ & 4 & 2,4 & 40,0 & \multirow[b]{2}{*}{0,94} & \multirow[b]{2}{*}{0,33} & \multirow[b]{2}{*}{0,05} \\
\hline & $>1948$ & 6 & 4,3 & 60,0 & & & \\
\hline
\end{tabular}

Fuente: Elaboración propia.

Según el análisis de la Tabla 6, la relación entre el año de nacimiento y la variable perfil profesionalidad es claramente significativa $(\mathrm{P} .=0,000)$, siendo esta una cualidad a la que los maestros nacidos posteriormente a 1948 aluden con mucha mayor frecuencia. Encontramos también que este grupo de maestros manifiesta en mucha menor medida la necesidad del perfil vocacional y de gusto por los niños para una labor docente satisfactoria que sus colegas nacidos antes de 1948. Otra diferencia que podemos destacar se refiere a la mayor necesidad de formación percibida por los docentes nacidos después de 1948.

Con respecto a las diferencias entre los maestros nacidos antes y después de 1948 y la variable requisitos formativos, Tabla 7 , hay que decir que la relación entre el año de nacimiento y la necesidad de formación en TIC, formación en metodologías prácticas, formación cultural y formación psicopedagógica es significativa $(\mathrm{P} .=0,000)$. Los resultados también evidencian que los maestros nacidos después de 1948 consideran más relevante la formación en TIC, en metodologías prácticas y en Psicopedagogía y, por el contrario, los nacidos antes de 1948 perciben como más importante la formación cultural.

\section{Discusión y conclusiones ¿Cómo debe ser un maestro del siglo XIX?}

Las claves para la sociedad y la economía del siglo XXI son la educación, el capital humano y la sociedad del conocimiento. Estos dos últimos factores, sin embargo, son siempre un producto del primero, lo que provoca que la educación pase a ser uno de los pilares básicos de la economía y ya no solo un derecho de los ciudadanos. Este papel, sin embargo, provoca que la planificación de la educación se haga, cada vez más, con objetivos sociales, por lo que existe el riesgo de dejar de lado los objetivos pedagógicos o que no se escuche suficientemente la voz de los maestros entre las de los políticos y los economistas (Esteve, 2014).

A este respecto, entre los maestros entrevistados, hay una diferencia significativa entre aquellos educados en el primer franquismo y los que crecieron con las reformas educativas del tardofranquismo y la democracia. Los nacidos antes de 1948 dan un rol más preponderante a la cultura y la disciplina en la educación, mientras que para los nacidos más adelante cobra más importancia la inclusión social, el uso de una buena metodología y la utilización de las Tecnologías de la Información y la Comunicación. La implantación de las nuevas tecnologías en las universidades y la lucha de la Unesco por establecer una comunidad científica abierta y global dan, si cabe, aún más importancia al dominio de los profesores sobre esta competencia clave, que debe capacitarles para trabajar en un entorno digital tanto de forma autónoma como colaborativa (Díaz Lázaro, 2014). Por otro lado, los maestros nacidos después de 1948 optan por un perfil más profesional de la labor docente como consecuencia de los cambios sociales acaecidos en las últimas décadas del siglo XX, así como por las dinámicas de cambio y transformación del sistema educativo al adaptarse a las exigencias de la sociedad actual. 
Asimismo, el perfil vocacional también muestra ciertas diferencias con respecto al género, mientras el 52,3\% de las mujeres dan mayor importancia a la vocación sobre otros factores, este es el caso solo en el 31,8 \% de los hombres. Entendemos que esta postura está muy relacionada con las salidas profesionales a las que hasta hace poco se veían limitadas las mujeres, especialmente en provincias donde los únicos estudios universitarios disponibles eran los de magisterio.

Teniendo todo esto en cuenta y con el objetivo de mejorar la competencia profesional del maestro, se han propuesto cuatro pilares fundamentales para la formación de los nuevos maestros: una sólida formación, una alta motivación, una elevada vocación y el compromiso firme con el progreso del alumno (Ministerio de Educación, Cultura y Deporte, 2015). Además, el nuevo docente del siglo XXI debe caracterizarse también por su compromiso social y por poseer una visión global (Bona, 2016).

Con este motivo, deben implantarse, en las facultades de Educación, programas que doten a los futuros maestros de las competencias esenciales para enfrentarse a su trabajo. Entre ellas no deben olvidarse la cultura general y el conocimiento de su materia; sin embargo, es esencial hacer un esfuerzo para incorporar varios conocimientos, que recogemos a continuación, cuya presencia los maestros entrevistados consideran todavía insuficiente.

A pesar de que las competencias pedagógicas y metodológicas, esenciales para un $47 \%$ de los maestros, sí están presentes en los planes educativos, la formación en estas competencias es insuficiente. Tres de cada cuatro profesores opinan que la falta de un componente práctico adecuado -orientado a la praxis en el aula- les ha provocado carencias en este ámbito en sus primeros años de profesión (Cantón-Mayo et al., 2013; Valle \& Manso, 2014).

En segundo plano quedan las competencias cultural y psicopedagógica $(35 \%$ y $30 \%$, respectivamente), también esenciales, pero que consideran que deben quedar supeditadas a las anteriores. Dentro de las competencias culturales, la competencia intercultural -es decir, la capacidad para mantener una interacción exitosa con una persona de otra cultura- ha adquirido una gran importancia, tanto para los maestros como para los estudiantes, con la consolidación del espacio común europeo. El cambio de paradigma en las fronteras ha creado nuevas necesidades laborales y la formación de los alumnos de nuestras escuelas y facultades de Educación es todavía, a todas luces, insuficiente en este ámbito (Sánchez Sánchez, 2014).

Por otra parte, destacan, aunque en este caso por la escasa importancia que les atribuyen los maestros, las competencias de gestión de centros y de resolución de situaciones de mediación (3,6\% y 3\% respectivamente). A pesar del sentir mayoritario de los maestros, consideramos que estas competencias no pueden olvidarse en la formación inicial, ya que su realidad laboral se las va a exigir en el futuro. De igual modo, las nuevas demandas sociales reclaman que los docentes asuman unas responsabilidades que tradicionalmente han recaído en la familia (Zapico-Barbeito et al., 2017): el desarrollo personal del alumno, el fomento de su autonomía, la formación de un espíritu crítico y la transmisión de unos valores y una cultura que la sociedad actual confía al maestro con el fin de que los transmita a los futuros ciudadanos.

En cuanto a las cualidades más importantes para el desempeño de su profesión, los maestros destacan la ilusión y la vocación (45\%). Al ser valores más difíciles de incluir en el currículo de los alumnos de magisterio -o más bien, para los que es muy complicado asegurar una correcta transmisión a pesar de los intentos de incluirlos en los programas educativos- llegamos a plantearnos si es posible enseñarlos. Si esto no fuera posible deberíamos decantarnos o bien por un proceso de selección a lo largo del periodo de formación, o bien por una selección en las pruebas de ingreso, para lo que deberían diseñarse instrumentos de medida que sean capaces de detectar si los futuros maestros atesoran las cualidades que su profesión les va a exigir. 
Con relación a la profesión de maestro contemplamos de manera imprescindible el esfuerzo por lograr convergencia entre norma, teoría y praxis educativa. Este es el reto que se le plantea a los centros de Educación, que deben ocuparse de formar a los futuros docentes en un momento en el que los valores tradicionales de la profesión de maestro han quedado relegados por la sociedad. En estos momentos de cambio se necesitan docentes que tengan dominio sobre las nuevas competencias, que sean capaces de formar ciudadanos participativos, críticos y responsables al servicio de una comunidad democrática. Al mismo tiempo, deben ejercer una docencia en la que se preste atención a la diversidad y que se dé de forma inclusiva.

De nuestras facultades de Educación debe surgir un profesor innovador, involucrado en la lucha por mejorar la calidad de la docencia, que destierre la individualidad en el ejercicio profesional y esté dispuesto para el trabajo colaborativo y la reflexión conjunta con otros docentes. En su desempeño no puede olvidar, tampoco, las competencias digitales y el uso de las TIC, que han llegado a las aulas para quedarse y que pueden tener un impacto mayor en la vida de nuestros estudiantes del que podemos ser conscientes en este momento.

No podemos olvidar, tampoco, el valor intrínseco del profesor como transmisor de valores. Su formación debe incorporar en todos los casos los principios de igualdad y equidad, que deben impregnar, tanto de forma implícita como explícita, todo el currículo educativo.

\section{Prospectiva ¿Dan respuesta las propuestas formativas a las necesidades de los futuros docentes?}

Hoy en día vivimos momentos similares a los que engendraron las reformas educativas de los años 70 y los 90 . Se acentúa el cambio de paradigma en el trabajo, ya iniciadoa finales del siglo pasado(Echeverría Samanes \& Martínez Clares, 2018). Por ello, las reformas educativas de principios del siglo XXI han seguido ese mismo camino, aunque, aportando una novedad muy importante. En el año 2006, la LOE introduce por primera vez en el sistema educativo el concepto de desarrollo competencial -información transferida a conocimiento- para hacer frente a las necesidades formativas de la sociedad actual. A partir de ese momento, tanto la reforma educativa del año 2013, LOMCE, como el actual texto de la Ley Orgánica de modificación de la LOE, LOMLOE, han ampliado y reforzado el alcance de esta formación en competencias, consideradas como elemento esencial curricular y sentando las bases para una educación para el siglo XXI.

Este paradigma de la profesión del magisterio, basado en la adquisición de nuevas competencias, nos conduce a deducir que para que los maestros sean capaces de transmitir competencias al alumnado deben haberlas adquirido e integrado durante su proceso de formación inicial y reforzado durante su proceso de formación continua.

A pesar de la importancia que la cultura normativa del siglo XXI le otorga al desarrollo competencial, y según las opiniones de una parte significativa de los docentes entrevistados, los procesos de enseñanza-aprendizaje que se dan en las facultades de Educación y en las escuelas universitarias de Magisterio todavía no han incorporado de forma suficiente estas competencias en sus estudiantes durante su proceso de formación inicial. Éstos, por su parte, tienen la sensación de verse obligados a desarrollarlas por su cuenta como parte de su praxis diaria. En síntesis, la formación recibida en la facultad no da respuesta a los nuevos retos que la sociedad plantea a los docentes, a los que ahora atribuye funciones sociales como la resolución de conflictos, la mediación con las instituciones educativas, la orientación y animación de los estudiantes o, incluso, en algunos casos, labores de asistencia social (Zapico-Barbeito et al., 2017). Por otra parte, las transformaciones que está operando la sociedad durante la pandemia también están generando un gran número de expectativas sobre los profesores, que deben enfrentarse a situaciones novedosas - medidas higiénicas, restricciones al contacto, traslado de las lecciones a espacios al aire libre- sin las herramientas adecuadas. 
Por todo lo anteriormente expuesto, no solo debemos formar docentes que sean capaces de enfrentarse a las actuales demandas sociales, sino que es nuestra responsabilidad darles las herramientas necesarias para que sean capaces de tener en cuenta el entorno social en el que van a desempeñar su labor, además de mejorar la conexión entre los contenidos y sus didácticas específicas (Valle \& Manso, 2014).

Es necesario, por tanto, hacer frente al reto que supone ampliar el alcance de la formación en competencias (digitales y tecnológicas, lingüísticas, matemática, sociales y de emprendimiento, las relacionadas con el trabajo cooperativo, etc.) planteada en las últimas reformas educativas. La implantación de esta educación basada en competencias requerirá un gran esfuerzo de adaptación metodológica en el que deben participar tanto las escuelas como las facultades. Es en este momento, en el que la sociedad se plantea transformar su sistema educativo, en el que los maestros y las maestras no deben dejarse llevar por el impulso, sino tomar las riendas del cambio para asegurarse de que la universidad y la experiencia educativa de los profesores mantienen su relevancia al establecerse las bases que guiarán el futuro de la educación (Arteaga Castillo \& Camargo, 2018).

A pesar de las dificultades detectadas, los maestros entrevistados consideran encomiable el esfuerzo de las facultades de Educación por mejorar su formación inicial, así como el trabajo en mejorar la formación didáctica y metodológica de los futuros profesores. Del mismo modo, destacan la importancia de la formación en competencias digitales y el trabajo con las Tecnologías de la Información y la Comunicación, con las que el profesor debe ser capaz de trabajar con unos alumnos que han crecido ya conectados al mundo digital. Con respecto a la formación continua, los maestros se decantan por una formación específica para cada materia. Además, señalan el gran valor que tiene para ellos el intercambio de experiencias con otros compañeros como parte de su proceso de formación continua.

Para concluir, nos gustaría señalar que los profesores involucrados en el estudio destacaron el valor de sentirse escuchados de nuevo, de participar en las nuevas propuestas metodológicas de aprendizaje, manteniendo en activo el conocimiento acumulado durante décadas de profesión. Es este un conocimiento secular que no podemos dejar escapar, que debemos recuperar y conservar, siendo conscientes de su tremenda fragilidad, para reintroducirlo en el sistema educativo, por ejemplo, a través del trabajo de investigación etnográfica de los alumnos de las facultades de educación. De este modo, estos se beneficiarán, asimismo, del contacto con experiencias reales de praxis docente, precisamente uno de los puntos débiles identificados por los maestros en su proceso de formación.

\section{Referencias}

Álvarez-López, G. (2015). La cualificación de maestro en Europa: Aportaciones a partir del análisis de las influencias supranacionales y los modelos europeos. Tendencias pedagógicas, 25, 9-34.

Arteaga Castillo, B., \& Camargo, S. (2018). Los sistemas educativos y la formación de maestros: Una relación compleja. Universidad Pedagógica Nacional.

Bolívar, A. (2014). Las historias de vida del profesorado: Voces y contextos. Revista Mexicana de Investigación Educativa, 62, 711-734.

Bona, C. (2016). Las escuelas que cambian el mundo. Círculo de Lectores.

Brady, A. M. (2020). From the reflective to the post-personal teacher. Teoria de la Educación. Revista Interuniversitaria, 32(1), 55-71.

Cantón-Mayo, I., Cañón, R., \& Arias, A. R. (2013). La formación universitaria de los maestros en Educación Primaria. Revista Interuniversitaria de Formación del Profesorado, 76 (27,1), 45-63.

De Gabriel Fernández, N. (2015). La profesionalización del magisterio en España (siglos XIX-XX): Literatura, biografías, memorialística. Historia y Memoria de la Educación, 1(1), 59-92. https://doi.org/10.5944/hme.1.2015.13232 
Díaz Lázaro, J. J. (2014). Metodologías colaborativas en el aula. Creación y diseño de recursos multimedia en red. In J. L. Alonso Roque, C. J. Gómez Carrasco, \& T. Izquierdo Rus (Eds.), La formación del profesorado en educación infantil y educación primaria (pp. 21-32). Universidad de Murcia.

Echeverría Samanes, B., \& Martínez Clares, P. (2018). Revolución 4.0, competencias, educación y orientación. Revista Digital de Investigación en Docencia Universitaria, 12(2), 4-34.

Esteve, J. M. (2014). La profesión docente ante los desafíos de la sociedad del conocimiento. In C. Vélaz de Medrano, \& D. Vaillant (Coords.), Aprendizaje y desarrollo profesional docente (pp. 17-29). OEI-Fundación Santillana.

Jaramillo, D. A., Cortés, E. J., \& Jaramillo, L. G. (2020). Experiencia, memoria y responsabilidad: La escuela como lugar de encuentro y donación. Teoría de la Educación. Revista Interuniversitaria, 32(1), $147-162$.

Llamas, F., \& Macías, E. (2018). Formación inicial de docentes en educación básica para la generación de conocimiento con las Tecnologías de la Información y la Comunicación. Revista Complutense de Educación, 29(2), 577-593.

Luzón Trujillo, A., \& Montes Moreno, S. (2018). Perspectiva histórica de la formación inicial del profesorado en España de Educación Infantil y Primaria. Una tarea inacabada. Historia Caribe, 13(33), 121-152.

Mainer Baqué, J., \& Mateos Montero, J. (2011). Saber, poder y servicio. Un pedagogo orgánico al servicio del Estado: Adolfo Maillo. Tirant lo Blanch.

Martín Fraile, B. (2015). La cultura escolar y el oficio de maestro. Educación XX1, 18(1), 147-166.

Martín Fraile, B. (2017). Testimonios de maestros: Modelos y prácticas. Ediciones Universidad de Salamanca.

Ministerio de Educación, Cultura y Deporte. (2015). El profesorado del siglo XXI. XXI Encuentro de Consejos Escolares Autonómicos y del Estado. Consejo Escolar del Estado. https://www.educacionyfp.gob.es/ dam/jcr:f88f1d40-af31-4fae-8c6b-fb5536f8ab71/21encuentroconsejosescolares2012-pdf.pdf

Prats, E. (2016). La formación inicial docente entre profesionalismo y vías alternativas: Mirada internacional. Bordón, 68(2), 19-33.

Romero-Martín, R., Castejón-Oliva, F.-J., López-Pastor, V.-M., \& Fraile-Aranda, A. (2017). Evaluación formativa, competencias comunicativas y TIC en la formación del profesorado. Comunicar, 52(25), 73-82. https://doi.org/10.3916/C52-2017-07

Sánchez Sánchez, G. (2014). Estudio sobre la competencia intercultural en la formación inicial del profesorado de educación primaria: El conocimiento sociocultural. In J. I. Alonso Roque, C. J. Gómez Carrasco, \& T. Izquierdo Rus (Eds.), La formación del profesorado en educación infantily educación primaria (pp. 325-336). Universidad de Murcia.

Tárraga Mínguez, R., Sanz-Cervera, P., Pastor Cerezuela, G., \& Fernández Andrés, M. I. (2017). Análisis de la autoeficacia percibida en el uso de las TIC de futuros maestros y maestras de Educación Infantil y Educación Primaria. Revista Electrónica Interuniversitaria de Formación del Profesorado, 20(3), 107-116.

Tiana, A. (2013). Los cambios recientes en la formación inicial del profesorado en España: Una reforma incompleta. Revista Española de Educación Comparada, (22), 39-58. https://doi.org/10.5944/reec.22.2013.9322

Valle, J. M. (2012). La política educativa supranacional: Un nuevo campo de conocimiento para abordar las políticas educativas en un mundo globalizado. Revista Española de Educación Comparada, (20), 109-144.

Valle, J. M., \& Manso, J. (2014). La voz del profesorado: Acceso a la profesión docente e inserción en el puesto de trabajo. Consejo General de Colegios de Doctores y Licenciados en Filosofía y Letras y en Ciencias.

Vega Gil, L. (1988). Las escuelas normales en Castilla y León, 1838-1900. Amarú Ediciones.

Zapico-Barbeito, M. E., Martínez, E., \& Montero M. L. (2017). Demandas sociales y formación inicial del profesorado: ¿Un callejón sin salida? Profesorado. Revista de currículum y formación del profesorado, 21(1), 80-102. 


\section{Disponibilidad de datos}

Los contenidos que subyacen al cuerpo de la investigación están contenidos en el artículo. Os dados subjacentes ao texto da pesquisa estão informados no artigo.

\section{Cómo citar este artículo}

Martín Fraile, B., Parra-Nieto, G., \& Rubio-Muñoz, F.-J. (2021). Pasado, presente y futuro de la formación de maestros en España. Cadernos de Pesquisa, 51, Artículo e08591. https://doi.org/10.1590/198053148591 